\title{
Trade-off in object versus spatial visualization abilities: Restriction in the development of visual-processing resources
}

\author{
Maria Kozhevnikov, Olesya Blazhenkova, and Michael Becker \\ George Mason University, Fairfax, Virginia
}

\begin{abstract}
Previous research indicates relative independence between the ventral and dorsal visual pathways, associated with object and spatial visual processing, respectively. The present research shows that, at the individualdifferences level, there is a trade-off, rather than independence, between object and spatial visualization abilities. Across five different age groups with different professional specializations, participants with above-average object visualization abilities (artists) had below-average spatial visualization abilities, and the inverse was true for those with above-average spatial visualization abilities (scientists). No groups showed both above-average object and above-average spatial visualization abilities. Furthermore, while total object and spatial visualization resources increase with age and experience, the trade-off relationship between object and spatial visualization abilities does not. These results suggest that the trade-off originates through a bottleneck that restricts the development of overall visualization resources, rather than through preferential experience in one type of visualization.
\end{abstract}

Neuroscience research demonstrates that visual areas of the brain divide into two distinct pathways: the object (ventral) and the spatial (dorsal) pathways (Courtney, Ungerleider, Keil, \& Haxby, 1996; Ungerleider \& Mishkin, 1982). The object pathway runs from the occipital lobe to the inferior temporal lobe, processing the visual appearances of objects. The spatial pathway runs from the occipital lobe to the posterior parietal lobe, processing spatial attributes.

Although anatomical and functional double-dissociation between the object and spatial systems has been demonstrated for visual perception and imagery (Farah, Hammond, Levine, \& Calvanio, 1998; Ungerleider \& Mishkin, 1982), this does not necessarily imply dissociation in individual differences in visualization. That is, object visualization ability (the ability to process visual information about objects or scenes in terms of color or shape) and spatial visualization ability (the ability to process information about spatial relations between objects or their parts and to perform spatial transformations) might be independent, but they also might tap the same underlying visualization ability. In the latter case, this underlying ability would not rely exclusively on object and/or spatial pathways, but would also depend on other brain areas involved in visual processing (e.g., prefrontal cortex, early visual areas).

Recently, neuroimaging research has suggested that object and spatial visualization abilities are distinct by showing differential activation of dorsal and ventral pathways, depending on one's spatial or object visualization ability. Whereas high spatial visualization ability was found to be associated with more efficient use of spatial-processing resources in the dorsal pathway, particularly in the right parietal cortex (Lamm, Bauer, Vitouch, \& Gstättner, 1999), high object visualization ability was associated with more efficient use of object processing resources in the ventral pathway, particularly in the lateral occipital complex (Motes, Malach, \& Kozhevnikov, 2008). In addition, participants with high spatial or object visualization ability demonstrated more efficient use of attentional areas (frontal and prefrontal regions) while solving spatial or object visualization tasks, respectively (Lamm et al., 1999; Motes et al., 2008). This suggests that both types of visualization ability, although distinct, might not be entirely independent, since both rely on common attentional resources (see also Miyake, Friedman, Rettinger, Shah, \& Hegarty, 2001).

Furthermore, Kozhevnikov, Kosslyn, and Shephard (2005) demonstrated that visualizers (individuals who prefer the use of visual over verbal processing) of high spatial ability succeeded on spatial visualization tasks (e.g., mental rotation) but performed poorly on object visualization assessments (e.g., generating pictorial images), whereas visualizers of high object ability showed the inverse profile. Consistent with the findings above, other studies have reported negative trends between the ability to generate and maintain pictorial images and the ability to perform spatial transformations (ranging from -0.30 to -0.44 ; Kosslyn, 
Brunn, Cave, \& Wallach, 1984; Poltrock \& Brown, 1984). Overall, these data suggest not only an object-spatial dissociation in individual differences, but also a trade-off between object and spatial visualization abilities.

The negative trend between the two types of visualization reported above remains puzzling. One possibility is that, throughout life, an individual experiences a long-term increase in efficacy in one visual strategy at the expense of the other. Many tasks can be solved by using either type of strategy: One may plan a route using a spatial map of an environment (spatial visualization) or by recalling a sequence of landmarks (object visualization). Therefore, it is possible that, for professionals whose training requires either spatial visualization, such as visualizing and dynamically transforming schematic images (scientists, engineers), or object visualization, such as attending to the visual appearances of scenes and objects (visual artists), the object-spatial trade-off gradually manifests from consistently exercising one type of visual processing over the other throughout educational training and professional practice. Indeed, research has shown that scientists have spatial skills superior to those of visual artists and that visual artists outperform scientists on tests that require the generation of high-resolution pictorial images (Casey, Winner, Brabeck, \& Sullivan, 1990; Kozhevnikov et al., 2005).

Another possibility is that the object-spatial visualization trade-off emerges due to limited-capacity shared resources, such as visual attention, which might be recruited by both types of visualization. That is, the limited capacity (bottleneck) of the visual attentional system might reduce the capacity of overall visualization resources. Thus, if an individual is genetically predisposed to either object or spatial visualization, this type of visualization might begin to develop at the expense of the other before any professional training, possibly during functional integration of the dorsal and ventral systems in early childhood. Then, not only adult professionals, but also children who have not received formal training but have been identified as gifted in one particular type of visualization, would exhibit below-average development of the other. If this were the case, once manifested, the trade-off should not be affected by amount of professional training.

The major goal of the present study was to demonstrate the trade-off between object and spatial visualization abilities and to examine whether the trade-off develops gradually with acquisition of visual expertise in a particular field or remains independent of age and experience. In particular, we investigated the relationship between the object and spatial visualization abilities of five different age groups of participants whose ages corresponded to their amount of specialized training, ranging from none or very little to extensive.

\section{Method}

Participants. The first two groups of participants consisted of "younger" $(10-13$ years of age; $n=76)$ and "older" (14-17 years of age; $n=123$ ) children from schools for the gifted in Moscow, Russia, and Teaneck, NJ. ${ }^{1}$ All were admitted to their schools on the basis of competitive exams and portfolio submissions evaluated by educational experts. The younger children had not yet received area-specific training, whereas the older children had. The third and fourth groups consisted of "younger" undergraduate (18-21 years of age; $n=69, M$ age $=20.09$ ) and "older" graduate and extended undergraduate $(22+$ years of age; $n=72, M$ age $=24.64)$ students from U.S. universities. The fifth group consisted of adult professionals $(n=73, M$ age $=29)$ who had college degrees and at least 2 years of professional experience in their fields. All the participants were recruited via advertisements posted in their schools, colleges, or professional communities.

On the basis of children's areas of giftedness (known from school memberships and concentrations), college students' majors, and adults' professional fields, each group was divided into three specialization subgroups: (1) science (physicists, mathematicians, computer scientists), (2) visual arts (painters, designers), and (3) humanities. In addition, there was a subgroup of generally gifted children, consisting of individuals who displayed high general intelligence without exclusive giftedness in any field, and a subgroup of adult architects. (Table 2 contains detailed information on participant demographics.)

Procedure. All the children and college students were administered three paper-and-pencil spatial visualization assessments - the Paper Folding Test (PFT), the Mental Rotation Test (MRT), and the spatial scale of the Object-Spatial Imagery and Verbal Questionnaire (OSIVQ) - and object visualization assessments: the Vividness of Visual Imagery Questionnaire (VVIQ), the Degraded Pictures Test (DPT), and the object scale of the OSIVQ. All the adult professionals received assessments similar to those given to the children and college students; however, the MRT and DPT were more complex, to suit experts better, and were computerized. In addition, they were administered the Grain Resolution Test (GRT), which required knowledge and abstract reckoning of specific objects' properties and, thus, was not suitable for the younger population. The order of the tests was randomized.

Materials. PFT (Ekstrom, French, \& Harman, 1976). The participants viewed successive drawings of folds made in a sheet of paper, with the last depicting a hole punched through the folded paper, and determined the pattern of holes in the paper if it were to be unfolded.

MRT (Shepard \& Metzler, 1971). The participants were shown three-dimensional forms, one of which was rotated relative to the other(s), and determined whether or not the figures were the same.

OSIVQ (Blazhenkova \& Kozhevnikov, 2009). The participants rated their agreement with statements describing object and spatial imagery preferences, abilities, and experiences.

VVIQ (Marks, 1973). The participants created mental images from verbal descriptions, and rated the images' vividness.

DPT (Kozhevnikov et al., 2005). The participants viewed degraded line drawings of common objects embedded in backgrounds of visual noise and identified the objects.

GRT (Kozhevnikov et al., 2005). The participants viewed a pair of names of objects, and determined which of the two objects had a denser grain, or particles/bits per unit area.

\section{Results}

Object-spatial trade-off within age groups. Composite spatial visualization scores $\left(Z_{\mathrm{spat}}\right)$ and composite object visualization scores $\left(Z_{\mathrm{obj}}\right)$ were created for each participant by averaging his/her $z$ scores (calculated separately for each age group) for all spatial and object assessments, respectively. ${ }^{2}$

The ANOVA results for $Z_{\mathrm{obj}}$ and $Z_{\mathrm{spat}}$ with specialization and gender as predictors are presented in Table 1 . The main effect of specialization was significant, with a large effect size for most groups. The main effect of gender was significant in younger (for $Z_{\text {spat }}$ ) and older (for $Z_{\mathrm{obj}}$ ) children, but not for students or professionals. The interaction between gender and specialization was significant only for $Z_{\text {spat }}$ for older college students. The effect sizes 
Table 1

ANOVA Results for $Z_{\text {obj }}$ and $Z_{\text {spat }}$ With Specialization and Gender As Predictors, Performed Separately for Each Age Group

\begin{tabular}{lllll}
\hline \multicolumn{1}{c}{ Groups } & $Z$ Scores & \multicolumn{1}{c}{ Specialization } & \multicolumn{1}{c}{ Gender } & \multicolumn{1}{c}{ Interaction } \\
\hline Children & $Z_{\text {obj }}$ & $F(3,75)=6.72^{* *}, \eta^{2}=.23$ & $F<1$ & $F(3,75)=1.05, \eta^{2}=.04$ \\
$\quad(10-13$ years $)$ & $Z_{\text {spat }}$ & $F(3,75)=5.20^{* *}, \eta^{2}=.19$ & $F(1,75)=4.88^{*}, \eta^{2}=.07$ & $F(3,75)=1.19, \eta^{2}=.05$ \\
Children & $Z_{\text {obj }}$ & $F(3,122)=2.25^{\dagger}, \eta^{2}=.06$ & $F(1,122)=7.21^{*}, \eta^{2}=.06$ & $F<1$ \\
$\quad(14-17$ years $)$ & $Z_{\text {spat }}$ & $F(3,122)=15.53^{* *}, \eta^{2}=.29$ & $F(1,122)=2.52, \eta^{2}=.02$ & $F<1$ \\
Students & $Z_{\text {obj }}$ & $F(2,68)=8.39^{* *}, \eta^{2}=.21$ & $F(1,68)=1.22, \eta^{2}=.21$ & $F(2,68)=1.19, \eta^{2}=.01$ \\
$\quad(M$ age $=20.09$ years $)$ & $Z_{\text {spat }}$ & $F(2,68)=8.76^{* *}, \eta^{2}=.22$ & $F(1,68)=1.76, \eta^{2}=.03$ & $F<1$ \\
Students & $Z_{\text {obj }}$ & $F(2,71)=20.23^{* *}, \eta^{2}=.38$ & $F(1,71)=2.86, \eta^{2}=.04$ & $F<1$ \\
$\quad(M$ age $=24.64$ years $)$ & $Z_{\text {spat }}$ & $F(2,71)=27.82^{* *}, \eta^{2}=.46$ & $F(1,71)=3.18^{\dagger}, \eta^{2}=.06$ & $F(2,71)=5.38^{*}, \eta^{2}=.14$ \\
Professionals & $Z_{\text {obj }}$ & $F(3,72)=15.06^{* *}, \eta^{2}=.41$ & $F(1,72)=2.21, \eta^{2}=.03$ & $F(3,72)=0.35, \eta^{2}=.02$ \\
& $Z_{\text {spat }}$ & $F(3,72)=9.81^{* *}, \eta^{2}=.31$ & $F(1,72)=0.46, \eta^{2}=.01$ & $F(3,72)=0.59, \eta^{2}=.03$ \\
\hline
\end{tabular}

${ }^{*} p<.05 . \quad{ }^{* *} p<.01 . \quad{ }^{\dagger} p<.1$.

of gender and interactions were small overall. The data are plotted in Figures 1A-1D for children and students and in Figure 2 for professionals.

In contrast to the classical speed-accuracy trade-off, in which an individual will naturally produce responses mutable along certain scales (accuracy, response time) but the relationship between the scales remains constant within the individual, the present study deals with a trade-off across subjects (Dennis \& Evans, 1996). In such a trade-off, an individual's responses are constant along two scales (e.g., scientists consistently perform high on spatial and low on object visualization tasks) but the relationship between these scales varies between individuals (e.g., artists consistently exhibit a pattern of responses that is the inverse of that for
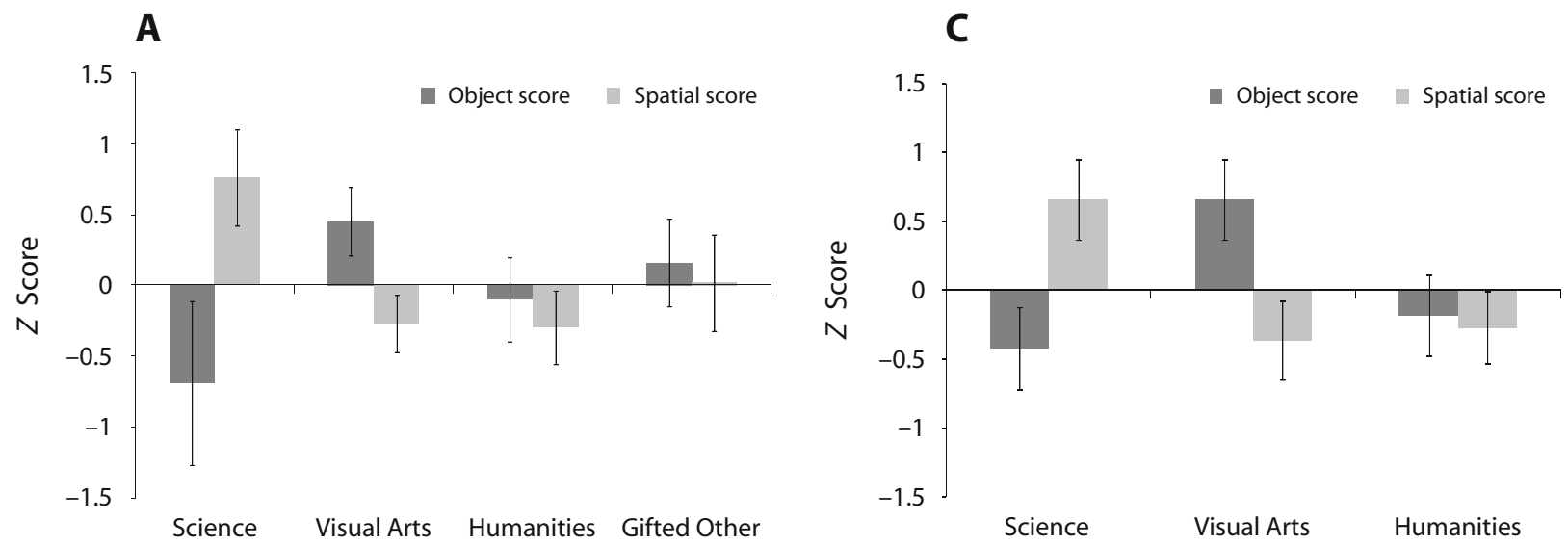

B

D
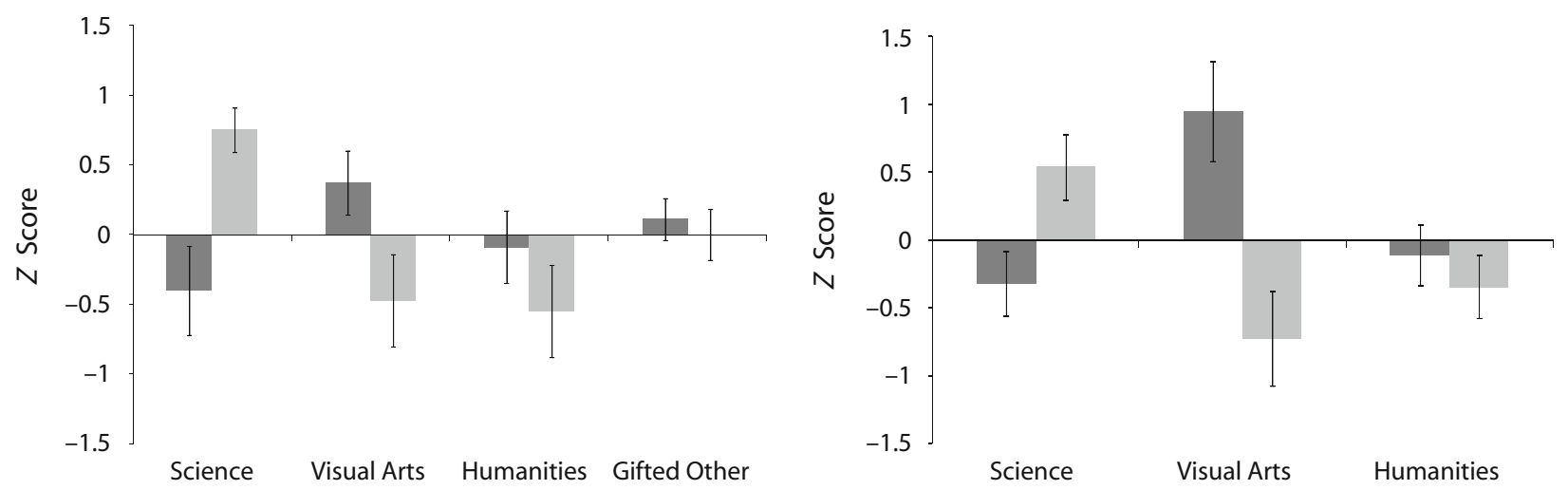

Figure 1. Normalized object and spatial visualization scores for different specialization subgroups of children and student participants. (A) Children, 10-13 years old. (B) Children, 14-17 years old. (C) Students, $M$ age $=20.09$ years. (D) Students, $M$ age $=$ $\mathbf{2 4 . 6 4}$ years. The bars represent $95 \%$ confidence intervals. 


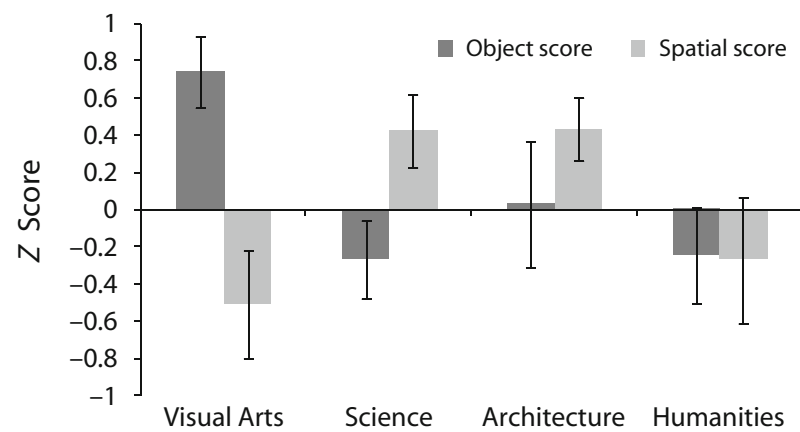

Figure 2. Normalized object and spatial visualization scores for different specialization subgroups of professionals. The bars represent $95 \%$ confidence intervals.

scientists). Because the relationship between the scales is different for different groups in the case of a trade-off across subjects, it cannot be fully represented by correlations. ${ }^{3}$ To demonstrate the existence of the object-spatial trade-off, it must be shown that no group scored above average on both object and spatial measures among the groups commonly described as having highly developed visualization abilities. The $95 \%$ confidence interval (Figures 1 and 2), which is the recommended technique for estimating the magnitude and direction of each group's difference from an estimated population average (Denis, 2003), revealed that groups specializing in the visual arts scored considerably above average on object visualization assessments, whereas their spatial scores were below average, and groups specializing in science demonstrated the inverse pattern. Finally, although the $Z_{\text {spat }}$ and $Z_{\mathrm{obj}}$ scores of architects and generally gifted children tended to be higher than or not different from average and the scores of those specializing in the humanities tended to be below or not different from average, no group showed both visualization abilities developed above average, supporting the existence of the trade-off between object and spatial visualization abilities (see Table 2 for $t$ test results).

The trade-off and overall visualization resources as a function of age and specialization. In order to compare the trade-off and its development across different age groups and specializations, we calculated $Z_{\text {obj }}$ and $Z_{\text {spat }}$ scores for the combined sample of children and students specializing in science, visual arts, and humanities (the generally gifted subgroup was not included, since it was absent in college students). ${ }^{4}$ Two separate scores for each participant were created. The first score, $Z_{\mathrm{obj}}+Z_{\mathrm{spat}}$, was created by adding $Z_{\text {obj }}$ and $Z_{\text {spat }}$ scores to reflect the overall amount of visualization (object and spatial) resources. The second score, $Z_{\mathrm{obj}}-Z_{\mathrm{spat}}$, was created by subtracting $Z_{\mathrm{spat}}$ from $Z_{\mathrm{obj}}$ to reflect the direction and magnitude of the trade-off between object and spatial visualization abilities (the absolute value of the $Z_{\mathrm{obj}}-Z_{\text {spat }}$ score reflects the magnitude of the trade-off; negative scores reflect development of spatial ability at the expense of object ability, and positive scores reflect the reverse). Figure 3 represents $Z_{\text {obj }}+Z_{\text {spat }}$ versus $Z_{\text {obj }}-Z_{\text {spat }}$ as a function of age.

An ANOVA conducted on the combined sample of children and students, with $Z_{\mathrm{obj}}+Z_{\mathrm{spat}}$ as a dependent variable and age, specialization, and gender as predictors, revealed a significant effect of specialization $[F(2,266)=7.76$, $\left.p<.001, \eta^{2}=.60\right]$. The $Z_{\mathrm{obj}}+Z_{\text {spat }}$ scores of groups of participants specializing in science and the visual arts were not significantly different ( $p=.64)$, but those specializing in the humanities had significantly lower $Z_{\mathrm{obj}}+Z_{\text {spat }}$ scores than did those specializing in science or the visual arts (all $p \mathrm{~s}<.001)$. There was a significant effect of age $\left[F(3,266)=2.98, p=.032, \eta^{2}=.035\right]$, indicating that the overall amount of visual-processing resources increases with age. The effect of gender and all the interactions were not significant $\left(F_{\mathrm{S}}<1\right)$.

In addition, ANOVAs conducted separately for $Z_{\text {spat }}$ and $Z_{\mathrm{obj}}$ as dependent variables, with age as a predictor, revealed that only $Z_{\text {spat }}$ significantly changes with age $\left[F(3,266)=5.94, p<.001, \eta^{2}=.064\right]$; specifically, it peaks in the age group of 14-17 years (during puberty) in both males $\left[F(3,115)=3.02, p=.033, \eta^{2}=.75\right]$ and females $\left[F(3,115)=3.45, p=.018, \eta^{2}=.066\right]$. Furthermore, the increase in $Z_{\text {spat }}$ is most prominent for the group of children specializing in science $[F(3,98)=4.66$,

Table 2

The Results of $t$ Tests for $Z_{\mathrm{obj}}$ and $Z_{\mathrm{spat}}$, As Compared With Average $(z=0)$, Performed Separately for Each Specialization and Age Group

\begin{tabular}{|c|c|c|c|c|c|c|}
\hline Groups & $Z$ Score & Visual Art & Science & Humanities & Generally Gifted & Architecture \\
\hline $\begin{array}{l}\text { Children } \\
\qquad(10-13 \text { years })\end{array}$ & $\begin{array}{c}\text { Total, females } \\
Z_{\text {obj }} \\
Z_{\text {spat }}\end{array}$ & $\begin{array}{c}21,18 \\
t(20)=3.97^{*} \\
t(20)=-9.73^{*}\end{array}$ & $\begin{array}{c}15,3 \\
t(14)=-3.53^{*} \\
t(14)=4.83^{*}\end{array}$ & $\begin{array}{c}21,11 \\
t(20)=-0.69 \\
t(20)=-2.37^{*}\end{array}$ & $\begin{array}{c}19,11 \\
t(18)=1.06 \\
t(18)=0.138\end{array}$ & \\
\hline $\begin{array}{l}\text { Children } \\
\text { (14-17 years) }\end{array}$ & $\begin{array}{c}\text { Total, females } \\
Z_{\text {obj }} \\
Z_{\text {spat }}\end{array}$ & $\begin{array}{c}19,17 \\
t(18)=3.42^{*} \\
t(18)=-3.06^{*}\end{array}$ & $\begin{array}{c}28,9 \\
t(27)=-2.51^{*} \\
t(27)=9.44^{*}\end{array}$ & $\begin{array}{c}22,15 \\
t(21)=-0.77 \\
t(21)=-3.46^{*}\end{array}$ & $\begin{array}{c}54,38 \\
t(53)=1.45 \\
t(53)=0.02\end{array}$ & \\
\hline $\begin{array}{l}\text { Students } \\
\qquad(M \text { age }=20.09 \text { years })\end{array}$ & $\begin{array}{c}\text { Total, females } \\
Z_{\text {obj }} \\
Z_{\text {spat }}\end{array}$ & $\begin{array}{c}21,17 \\
t(20)=4.74^{*} \\
t(20)=-2.61^{*}\end{array}$ & $\begin{array}{c}22,8 \\
t(21)=-2.96^{*} \\
t(21)=4.77^{*}\end{array}$ & $\begin{array}{c}26,24 \\
t(25)=-1.26 \\
t(25)=-2.08^{*}\end{array}$ & & \\
\hline $\begin{array}{l}\text { Students } \\
\qquad(M \text { age }=24.64 \text { years })\end{array}$ & $\begin{array}{c}\text { Total, females } \\
Z_{\text {obj }} \\
Z_{\text {spat }}\end{array}$ & $\begin{array}{c}14,6 \\
t(13)=5.43^{*} \\
t(13)=-4.37^{*}\end{array}$ & $\begin{array}{c}34,13 \\
t(23)=-2.73^{*} \\
t(23)=4.79^{*}\end{array}$ & $\begin{array}{c}24,10 \\
t(23)=-0.99 \\
t(23)=-3.09^{*}\end{array}$ & & \\
\hline Professionals & $\begin{array}{c}\text { Total, females } \\
Z_{\text {obj }} \\
Z_{\text {spat }}\end{array}$ & $\begin{array}{c}16,8 \\
t(15)=8.48^{* *} \\
t(15)=-3.86^{*}\end{array}$ & $\begin{array}{c}24,6 \\
t(23)=-2.54^{*} \\
t(23)=4.61^{* *}\end{array}$ & $\begin{array}{c}23,14 \\
t(22)=-2.01^{\dagger} \\
t(22)=-1.66\end{array}$ & & $\begin{array}{c}10,2 \\
t(9)=0.22 \\
t(9)=4.74^{*}\end{array}$ \\
\hline
\end{tabular}

${ }^{*} p<.05 . \quad{ }^{* *} p<.01 . \quad \dagger p<.1$. 


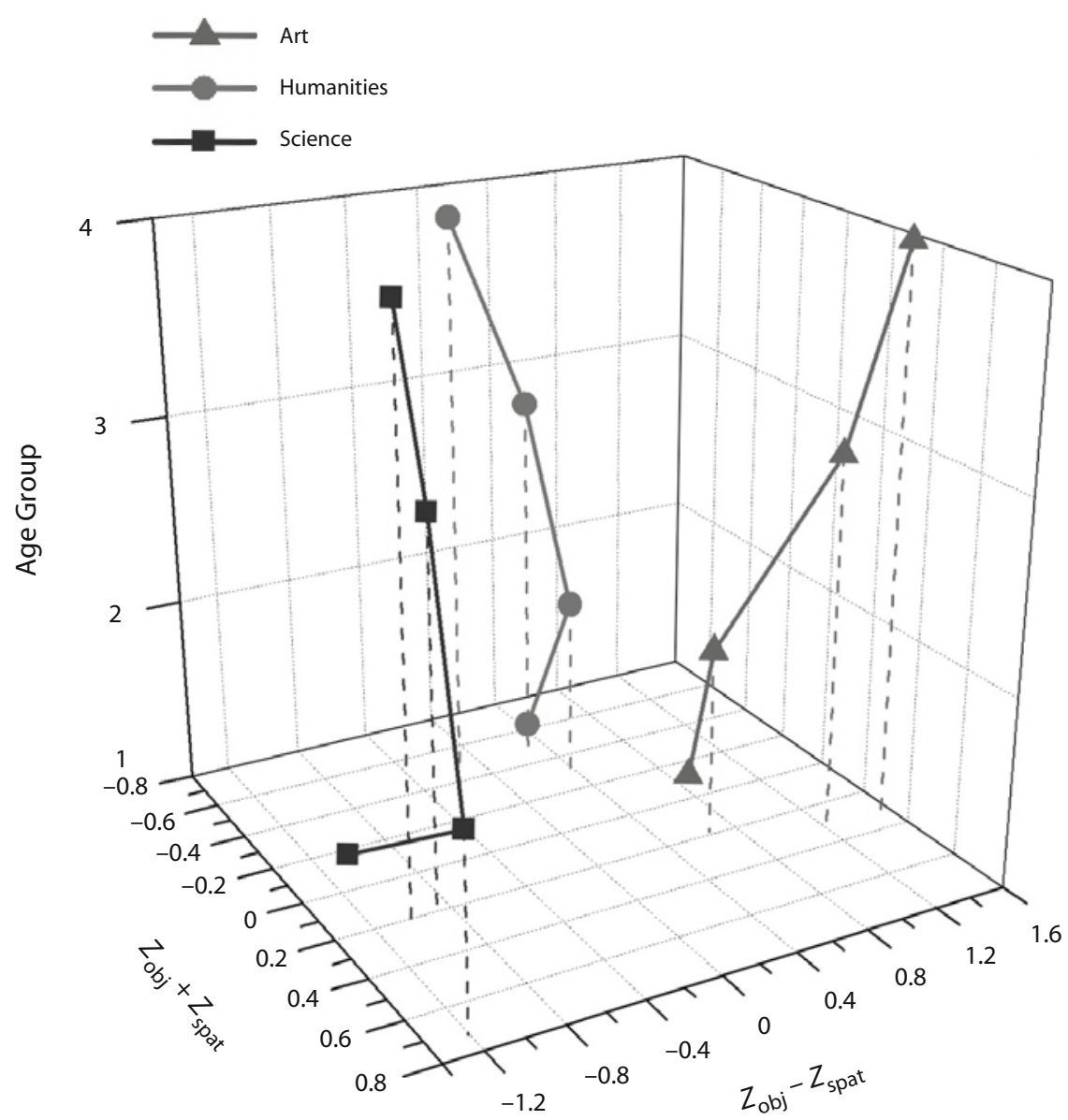

Figure 3. $Z_{\mathrm{obj}}+Z_{\text {spat }}$ versus $Z_{\mathrm{obj}}-Z_{\text {spat }}$ for different specialization subgroups and age groups. (1) Children, 10-13 years old. (2) Children, 14-17 years old. (3) Students, $M$ age = 20.09 years. (4) Students, $M$ age $=24.64$ years.

$\left.p=.015, \eta^{2}=.104\right]$ but is not significant for those with interests in the humanities or the visual arts (all $F \mathrm{~s}<1)$. Although there was no overall significant increase in $Z_{\mathrm{obj}}$ with age in either gender (all $F_{\mathrm{s}}<1$ ), the increase in $Z_{\mathrm{obj}}$, during the transition from the age of 14-17 years to that of 18-21 years, was significant for those specializing in the visual arts $\left[F(3,74)=4.06, p<.01, \eta^{2}=.146\right]$ and not significant for those specializing in science or the humanities (all $F \mathbf{s}<1$ ).

These results are consistent with those of other developmental studies suggesting that spatial resources fully mature at the end of middle childhood (ages 14-17 years), whereas object resources continue to develop gradually throughout adolescence (Van Leijenhorst, Crone, \& Van der Molen, 2007). However, our data extend the previous findings to indicate that the significant increase in spatial visualization ability at the age of $14-15$ years occurs only in the group of children specializing in science, whereas no such increase was observed in the other children. Similarly, the only significant increase in object visualization ability occurs around the age of 18-21 years in the group of visual art majors.

An ANOVA with $Z_{\mathrm{obj}}-Z_{\text {spat }}$ as a dependent variable and age, specialization, and gender as predictors revealed a highly significant effect for specialization $[F(2,266)=$ 84.17, $\left.p<.001, \eta^{2}=.41\right]$. There were significant differences in $Z_{\mathrm{obj}}-Z_{\mathrm{spat}}$ between the groups (all $p \mathrm{~s}<.001$ ), with the trade-off favoring spatial visualization ability in those specializing in science and object visualization ability in those specializing in the arts, and with no trade-off (object and spatial visualization abilities developed more or less equally, but not above average) in those specializing in the humanities. The effect of gender was also significant, but the effect size was small $\left(\eta^{2}=.08\right)$. The effect of age was not significant $\left[F(3,266)=2.47, p=.63, \eta^{2}=\right.$ $.03]$, nor were the interactions, indicating that the magnitude and direction of the trade-off do not change with age and corresponding amount of professional experience.

The trade-off and overall visualization resources as a function of specialization in adult professionals. Similar analyses were performed on the sample of adult professionals. An ANOVA with $Z_{\mathrm{obj}}+Z_{\mathrm{spat}}$ as the dependent variable and specialization and gender as predictors revealed a significant effect of specialization $[F(3,72)=$ $\left.4.67, p=.005, \eta^{2}=.18\right]$. No other effects were significant. Only humanities professionals have a significantly lower $Z_{\mathrm{obj}}+Z_{\mathrm{spat}}$ score than do the other groups ( $p<$ .05 ). The other three groups (visual artists, scientists, and 
architects) did not show any significant differences ( $p>$ .74). As for the $Z_{\mathrm{obj}}-Z_{\mathrm{spat}}$ score, significant differences were found between the groups $[F(3,72)=21.63, p<$ $\left..001, \eta^{2}=.50\right]$, indicating the largest magnitude of tradeoff in scientists (favoring spatial ability) and visual artists (favoring object ability) and the smallest in architects and humanities professionals (for multiple-group comparisons, all $p \mathrm{~s}<.01$, except humanities professionals vs. architects, who were not significantly different). Figure 4 represents $Z_{\mathrm{obj}}+Z_{\text {spat }}$ versus $Z_{\mathrm{obj}}-Z_{\text {spat }}$ for adult professionals.

\section{Discussion}

The results provide support for the existence of a trade-off between object and spatial visualization abilities. Analyses from all samples revealed the same pattern: Those who specialize in the visual arts have above-average object visualization skills and below-average spatial visualization ability, and the opposite pattern is seen in those who specialize in science. None of the groups showed both above-average spatial and above-average object visualization skills. Furthermore, within each age group, those specializing in different visual fields (art, science, and architecture) demonstrated the same high but finite amount of total visual-processing resources, which can be differentially distributed to object or spatial visualization ability or some combination of the two.

It is not surprising that the gifted children 10-13 years of age already exhibited one visualization type developed above average. This could be explained by innate predispositions of these children to one type of visualization, which usually manifest before deliberate practice and training (Winner, 1996). It is also possible that a child who displays a proficiency in one type of visualization early in life comes from parents who are also genetically predisposed to that particular form of visualization and would probably guide and develop that visualization type in their children at very early ages. What is surprising, however, is that these gifted children already displayed below-average development of the other type of visualization and that the direction and magnitude of the trade-off does not depend on education or professional training.

Thus, the findings above support the possibility that the trade-off originates through a bottleneck that restricts the development of overall visualization resources, rather than through preferential experience in one type of visualization. Visual attention is indeed critical for both spatial processing, such as maintaining information about spatial locations and performing spatial transformations (Awh \& Jonides, 2001), and object processing, such as binding featural information in memory and selecting perceptual objects (Wheeler \& Treisman, 2002). The prefrontal cortex, associated with visual attention, has reciprocal connections with both the temporal and parietal cortices and acts as a source of biasing activity in these areas (Desimone, 1996). Because of capacity limitations of visual attention, a child with high spatial-processing resources, for instance, may consistently attend to spatial representations and locations throughout the course of his or her cognitive development, at the expense of attending to perceptual objects and their features. Early in life (possibly during functional integration of the dorsal and ventral pathways), this preferential attention might favor the development of spatial-processing abilities through more consistent use of spatial pathways, while restricting the development of object-processing abilities due to long-term underuse of object pathways. The snowballing effect appears to further diverge later in scientists (ages, 14-17 years) and artists (ages, 18-21 years) during the critical period in the development of their corresponding type of visualization ability. That is, only those who have a predisposition for a particular visualization ability demonstrate a significant increase in this type of visualization during a certain age. However, further research is needed to verify the above,

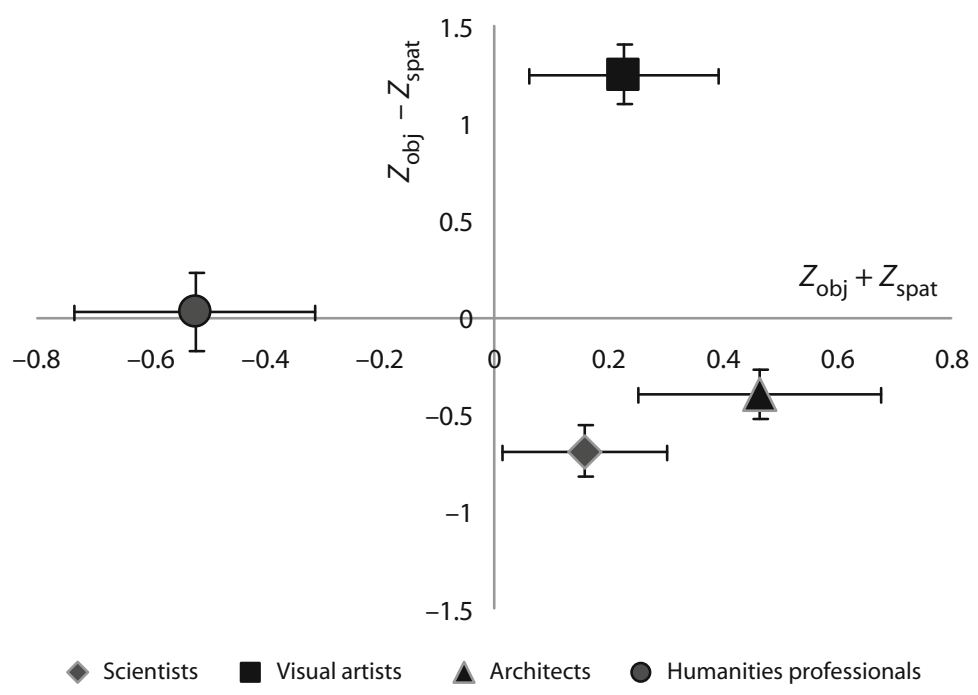

Figure 4. $Z_{\mathrm{obj}}+Z_{\text {spat }}$ versus $Z_{\mathrm{obj}}-Z_{\text {spat }}$ scores for different specialization subgroups of professionals. The bars represent $\pm 1 S E M$. 
due to limitations of our cross-sectional sampling. Longitudinal research from infancy to early childhood examining the changes in the capacity of attentional, as well as object and spatial, resources could ultimately explain the origins of the observed trade-off.

Research has also shown that gender might also influence the direction of the trade-off (object ability tends to surpass spatial ability in females, and the opposite is true for males) but that its effect is secondary to that of specialization, inconsistent across samples, relatively small, and reduced in adult professionals.

Since our data indicate that object and spatial visualization abilities might not develop entirely independently in those with high ability and talent in a visual professional field, one important future research direction would be to investigate how specialized education might foster different types of visualization and what the best age would be to start specialized training that builds on early-age predispositions. Finally, our results have important implications for neuroscience research, suggesting an interaction between attentional resources and visualization abilities and shedding light on how visual processing might differ between individuals.

\section{AUTHOR NOTE}

The research was supported by the Office of Naval Research under Grants ONR-N000140810382 and ONR-N000140611072 to M.K. We thank Eric Nordheim and Patrick McKnight for their statistical advice. Correspondence concerning this article should be addressed to M. Kozhevnikov, Department of Psychology, George Mason University, 4400 University Drive, MSN 3F5, Fairfax, VA 22030 (e-mail: mkozhevn@ gmu.edu).

\section{REFERENCES}

Awh, E., \& Jonides, J. (2001). Overlapping mechanisms of attention and working memory. Trends in Cognitive Sciences, 5, 119-126.

Blazhenkova, O., \& Kozhevnikov, M. (2009). The new objectspatial-verbal cognitive style model: Theory and measurement. $A p-$ plied Cognitive Psychology, 23, 638-663.

Casey, M. B., Winner, E., Brabeck, M., \& Sullivan, K. (1990). Visual-spatial abilities in art, math, and science majors: Effects of sex, handedness, and spatial experience. In K. Gilhooly, M. Keane, R. Logie, \& G. Erdos (Eds.), Lines of thinking: Reflections on the psychology of thought (Vol. 2, pp. 275-249). New York: Wiley.

Courtney, S. M., Ungerleider, L. G., Keil, K., \& Haxby, J. V. (1996). Object and spatial visual working memory activate separate neural systems in human cortex. Cerebral Cortex, 6, 39-49.

DENIS, D. J. (2003). Alternatives to null hypothesis significance testing. Theory \& Science, 4, 1-22.

Dennis, I., \& Evans, J. ST. B. T. (1996). The speed-error trade-off problem in psychometric testing. British Journal of Psychology, 87, 105-129.

Desimone, R. (1996). Neural mechanisms for visual memory and their role in attention. Proceedings of the National Academy of Sciences, 93, 13494-13499.

Ekstrom, R. B., French, J. W., \& Harman, H. H. (1976). Kit of factor-referenced cognitive tests. Princeton, NJ: Educational Testing Service.

Farah, M. J., Hammond, K. M., Levine, D. N., \& Calvanio, R. (1998). Visual and spatial mental imagery: Dissociable systems of representation. Cognitive Psychology, 20, 439-462.

Kosslyn, S. M., Brunn, J., Cave, K. R., \& Wallach, R. W. (1984). Individual differences in mental imagery ability: A computational analysis. Cognition, 18, 195-243.

Kozhevnikov, M., Kosslyn, S., \& Shephard, J. (2005). Spatial versus object visualizers: A new characterization of visual cognitive style. Memory \& Cognition, 33, 710-726.

Lamm, C., Bauer, H., Vitouch, O., \& Gstättner, R. (1999). Differences in the ability to process a visuo-spatial task are reflected in event-related slow cortical potentials of human subjects. Neuroscience Letters, 269, 137-140.

MARKs, D. F. (1973).Visual imagery differences in the recall of pictures British Journal of Psychology, 64, 17-24.

Miyake, A., Friedman, N. P., Rettinger, D. A., Shah, P., \& HeGARTY, M. (2001). How are visuospatial working memory, executive functioning, and spatial abilities related? A latent-variable analysis. Journal of Experimental Psychology: General, 130, 621-640.

Motes, M. A., Malach, R., \& Kozhevnikov, M. (2008). Objectprocessing neural efficiency differentiates object from spatial visualizers. NeuroReport, 19, 1727-1731.

Poltrock, S. E., \& Brown, P. (1984). Individual differences in visual imagery and spatial ability. Intelligence, 8, 93-138.

Shepard, R. N., \& Metzler, J. (1971). Mental rotation of threedimensional objects. Science, 171, 701-703.

UngerLeider, L. G., \& Mishrin, M. (1982). Two cortical visual systems. In D. J. Ingle, M. A. Goodale, \& R. J. W. Mansfield (Eds.), Analysis of visual behavior (pp. 549-586). Cambridge, MA: MIT Press.

Van Leijenhorst, L., Crone, E. A., \& Van der Molen, M. W. (2007). Developmental trends for object and spatial working memory: A psychophysiological analysis. Child Development, 78, 987-1000.

Wheeler, M. E., \& Treisman, A. M. (2002). Binding in short-term visual memory. Journal of Experimental Psychology: General, 131, 48-64.

Winner, E. (1996). Gifted children: Myths and realities. New York: Basic Books.

\section{NOTES}

1. The study was primarily conducted in Russia because of its specialized schools and competitive admissions system for gifted children; the school in Teaneck, NJ, has a similar competitive admissions system.

2. To support the legitimacy of creating the combined scores, we conducted a factor analysis on children and students, which revealed, consistent with previous results (Blazhenkova \& Kozhevnikov, 2009), two distinct factors, with all spatial assessments loaded onto one factor $(.590, .798, .840)$ and all object assessments loaded onto the other $(.509$, $.793, .840)$

3. Indeed, the correlations between $Z_{\mathrm{obj}}$ and $Z_{\mathrm{spat}}$ are sensitive to individuals' specialization. The correlation between $Z_{\text {obj }}$ and $Z_{\text {spat }}$ tends to be negative $(r=-.17, p=.17$, in professionals; $r=-.10, p=.06$, in children and college students combined), but it becomes highly significant if humanities specialists are excluded from the analysis $(r=-.42$, $p=.003$, in professionals; $r=-.20, p=.002$, in children and college students combined).

4. Adult professionals were not included in this analysis, due to the differences in the more advanced test versions administered to them.

(Manuscript received May 15, 2009; revision accepted for publication September 18, 2009.) 